\title{
Influence of nanoparticle shapes on natural convection flow with heat and mass transfer rates of fractional nanofluids
}

\author{
K.R. Madhura ${ }^{1}$, Babitha Atiwale ${ }^{2}$, and S.S. Iyengar ${ }^{3}$ \\ ${ }^{1}$ The National College \\ ${ }^{2}$ East West Institute of Technology \\ ${ }^{3}$ Florida International University
}

October 29, 2020

\begin{abstract}
This article presents an investigation on free convection flow and heat and mass transfer of fractional nanofluids formed by different base fluids with different shaped copper nanoparticles past an infinite vertical plate with radiation effect. The model which describes the system involving partial differential equations is converted into model of fractional nanofluids using Caputo time derivatives, which explains the impact of memory on behavior of nanofluids and are solved by employing technique of Laplace transform. The different base fluids - water $\$\left(\mathrm{H}_{-} 2 \mathrm{O}\right) \$, \$ S A \$$ :sodium alginate $\$\left(\mathrm{C}_{-}\{6\} \mathrm{H}_{-}\{9\} \mathrm{Na}_{-} \mathrm{O}_{-}\{7\}\right) \$$ and $\$ \mathrm{EG} \$$ :ethylene glycol $\$\left(\mathrm{C}_{-}\{2\} \mathrm{H}_{-}\{6\} \mathrm{O}_{-}\{2\}\right) \$$ and various shapes of nanoparticles - blade, brick, platelet and cylinder are considered for the study. The exact solutions are obtained for the temperature, concentration and velocity distributions and the respective Nusselt number, Sherwood number and skin-friction coefficient. The influence of non-dimensional parameters like fractional parameter, Schmidt number, radiation parameter, nanoparticle volume fraction, etc., which provides physical interpretations of temperature, concentration and velocity fields, Nusselt number, Sherwood number and skin-friction are discussed in detail with the aid of graphical representations. From the results it is found that fractional nanofluids formed by water based blade shaped nanoparticle exhibits more velocity and temperature distributions. Also, strengthen of fluid flow, temperature and concentration of nanofluids are inversely correlate with the order of fractional derivatives.
\end{abstract}

\section{Hosted file}

Dr.K.R.Madhura.pdf available at https://authorea.com/users/371209/articles/489633-influenceof-nanoparticle-shapes-on-natural-convection-flow-with-heat-and-mass-transfer-rates-offractional-nanofluids 
figures/flow/flow-eps-converted-to.pdf 
figures/temphi/temphi-eps-converted-to.pdf 
figures/tempshape/tempshape-eps-converted-to.pdf 
figures/tempbase/tempbase-eps-converted-to.pdf 
figures/consc/consc-eps-converted-to.pdf 
figures/velphi/velphi-eps-converted-to.pdf 
figures/velshape/velshape-eps-converted-to.pdf 
figures/velbase/velbase-eps-converted-to.pdf 
figures/nubaser0/nubaser0-eps-converted-to.pdf 
figures/nubaser1/nubaser1-eps-converted-to.pdf 
figures/nushaper0/nushaper0-eps-converted-to.pdf 
figures/nushaper1/nushaper1-eps-converted-to.pdf 
figures/sherwood/sherwood-eps-converted-to.pdf 
figures/skinbaser0/skinbaser0-eps-converted-to.pdf 
figures/skinbaser1/skinbaser1-eps-converted-to.pdf 
figures/skinshaper0/skinshaper0-eps-converted-to.pdf 
figures/skinshaper1/skinshaper1-eps-converted-to.pdf 